\title{
Yield Ratios in Particle- $\gamma$ Coincidence as a Spectroscopic Tool
}

\author{
K. T. Wiedemann, N. H. Medina, J. R. B. Oliveira, J. A. Alcántara-Núñez, \\ W. A. Seale, R. V. Ribas, E. W. Cybulska, and M.A.G. Silveira \\ Instituto de Física da Universidade de São Paulo, São Paulo, SP, Brazil
}

Received on 1st December, 2004

\begin{abstract}
Two nuclear reactions, ${ }^{11} \mathrm{~B}+{ }^{100} \mathrm{Mo},\left(\mathrm{E}_{b}=43 \mathrm{MeV}\right)$ and ${ }^{16} \mathrm{O}+{ }^{51} \mathrm{~V},\left(\mathrm{E}_{b}=70 \mathrm{MeV}\right)$ were studied with a $\gamma$ spectrometer with a charged particle array in order to measure the correlation between $\gamma$-rays emitted by residual nuclei produced by heavy-ion reactions in coincidence with a charged particles detected at different angles. Ratios of $\gamma$-transition intensities in coincidence with charged particles were measured for the main products of these reactions, providing a differentiation between the exit channels, even when complete fusion is the only reaction mechanism.
\end{abstract}

\section{INTRODUCTION}

Fusion-evaporation reactions have been used in the production of nuclei at high angular momentum and excitation energies. The production cross-section for the residual nuclei produced by this reaction mechanism are usually well described by the Monte Carlo code PACE (Projection Angular Momentum Coupled Evaporation)[1]. However, these predictions are not in good agreement with the experimental results when the nuclei are populated by other reaction mechanisms, such as incomplete fusion. In the fusion process of the projectile and target nuclei, it is possible that one of them breaks up into two or more nucleonic clusters prior to the formation of a completely fused system. When the projectile breaks up into two clusters (break-up process) and the fusion of one of them and the target occurs, this mechanism is referred to as incomplete fusion. In this case, there is an anisotropy in the angular distribution of the emitted particles.

Dracoulis et al.[2] suggested that the presence of incomplete fusion, enhancing the anisotropy in the angular distribution of emitted particles, produces different intensities of $\gamma$-transitions when in coincidence with particles detected at backward and forward angles (yield ratios) for the isotopes formed in a heavy-ion reaction. In this work, this hypothesis is investigated with the determination of these yield ratios as a function of the $\gamma$-ray energy. The yield ratios were analyzed for eight isotopes produced in the ${ }^{11} \mathrm{~B}+{ }^{100} \mathrm{Mo}$ and ${ }^{16} \mathrm{O}+{ }^{51} \mathrm{~V}$ reactions.

\section{EXPERIMENTAL PROCEDURES}

Two reactions were measured at the Pelletron Accelerator of the Universidade de São Paulo: ${ }^{11} \mathrm{~B}+{ }^{100} \mathrm{Mo}\left(E_{b}=\right.$ $43 \mathrm{MeV}$ ), with a target of a $16 \mathrm{mg} / \mathrm{cm}^{2}$ foil of ${ }^{100} \mathrm{Mo}$, and ${ }^{16} \mathrm{O}+{ }^{51} \mathrm{~V}\left(E_{b}=70 \mathrm{MeV}\right)$, with a target of a $4.4 \mathrm{mg} / \mathrm{cm}^{2}$ foil of ${ }^{51} \mathrm{~V}$ on an evaporated $7 \mathrm{mg} / \mathrm{cm}^{2}$ gold backing. The SaciPerere array[3] was used to measure a correlation of $\gamma$-rays in coincidence with charged particles. The Saci (Sistema Ancilar de Cintiladores) is a $4 \pi$ charged particle telescope system consisting of 11 plastic phoswich scintillators disposed in the geometry of a dodecahedron. This system enables the selection of the charged particle fold in coincidence with the observed $\gamma$-rays. The charged particle detectors were screened against the scattered heavy ions with aluminum foils. Perere (Pequeno Espectrômetro de Radiação Eletromagnética com Rejeição de Espalhamento) is the $\gamma$-ray spectrometer consisting of 4 HPGe detectors with BGO Compton-shields. The two fusion reactions were performed mainly for nuclear structure studies, with special interest in the nuclei: ${ }^{105} \mathrm{Rh}[4]$, ${ }^{108} \mathrm{Pd}[5]$ and ${ }^{60,61} \mathrm{Ni}[6] . \gamma \gamma$ and $\gamma$-particle matrices in coincidence with protons and $\alpha$-particles detected at forward $\left(0^{\circ}\right)$, middle $\left(63^{\circ}\right)$ and backward $\left(117^{\circ}\right)$ angles were analyzed in order to extract yield ratios between the $\gamma$-transition intensities.

\section{DATA ANALYSIS AND EXPERIMENTAL RESULTS}

\section{a) The ${ }^{11} \mathbf{B}+{ }^{100}$ Mo reaction}

The experimental cross-section for the production of the ${ }^{105} \mathrm{Rh}$ nucleus in the reaction ${ }^{11} \mathrm{~B}+{ }^{100} \mathrm{Mo}$ is about four times larger than estimated by the code PACE, indicating a significant contribution of another mechanism in the production of this nucleus.

In Figure 1 the yield ratios in rhodium and palladium products are shown. In Figure $1 \mathrm{a}$ it is not possible to differentiate the yield ratios for different palladium isotopes $\left({ }^{107} \mathrm{Pd}\right.$ and ${ }^{108} \mathrm{Pd}, p 3 n$ and $p 2 n$ channel, respectively), which indicates an isotropic distribution of emitted particles. Nevertheless, it is possible to see in Figure 1b different yield ratios for selected transitions in rhodium products: ${ }^{104} \mathrm{Rh}$ and ${ }^{105} \mathrm{Rh}(\alpha 3 n$ and $\alpha 2 n$ channel, respectively), suggesting that the difference is due to the presence of incomplete fusion.

The $\gamma$-transition of $216 \mathrm{keV}$ is an intense peak in the $\gamma$-ray spectrum, related with both ${ }^{105} \mathrm{Rh}$ and ${ }^{104} \mathrm{Rh}$. The yield ratio indicates that its intensity may be predominantly attributed to ${ }^{104} \mathrm{Rh}$.

In Figure 2 the $\alpha$-particle spectra in coincidence with ${ }^{104} \mathrm{Rh}$ and ${ }^{105} \mathrm{Rh} \gamma$-rays are shown, indicating higher energy $\alpha$ particles associated with ${ }^{105} \mathrm{Rh}$. The presence of these higher energy $\alpha$-particles supports the hypothesis of a break-up process, typically related with incomplete fusion mechanism.

Taking into account the experimental conditions, yield ratios were simulated using the particle energy spectra calculated by the code PACE, since the number of detected particles 

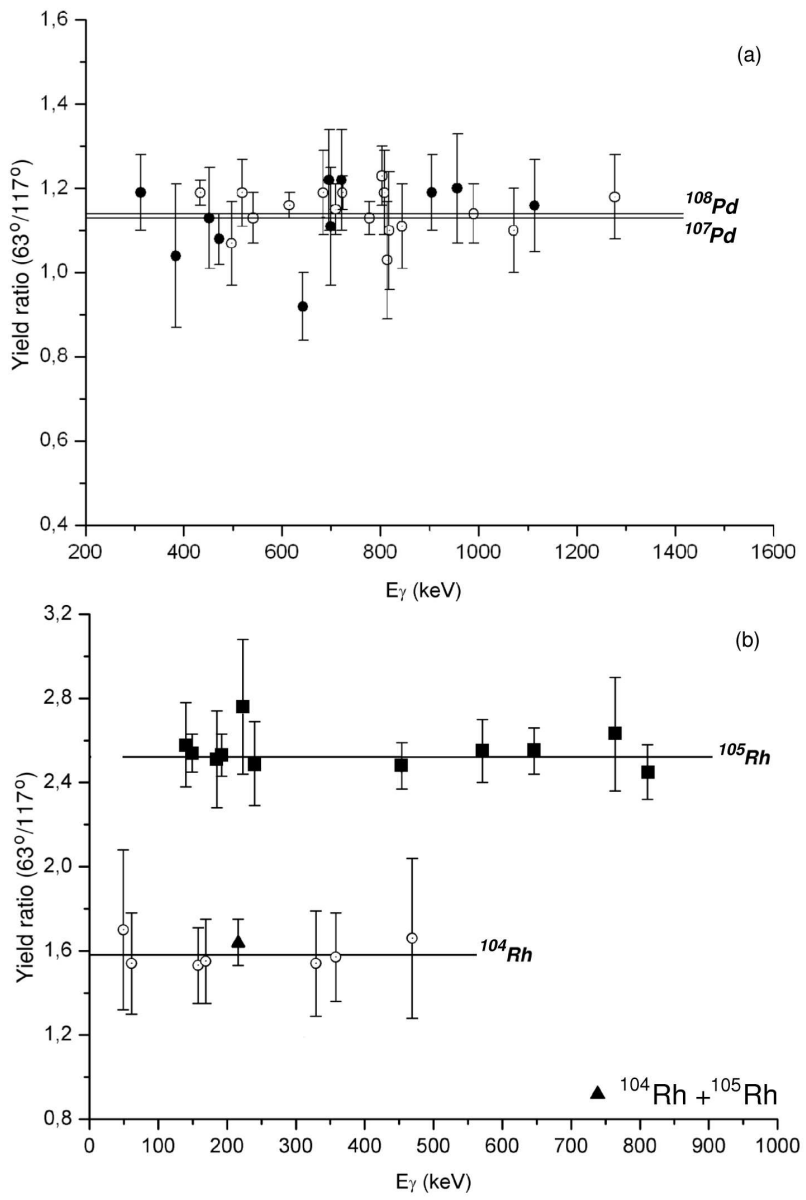

FIG. 1: The yield ratios for selected $\gamma$-transitions (in coincidence with particles detected at $63^{\circ}$ and $117^{\circ}$ ), in palladium (a) and in rhodium products (b), as a function of $\gamma$-ray energy.

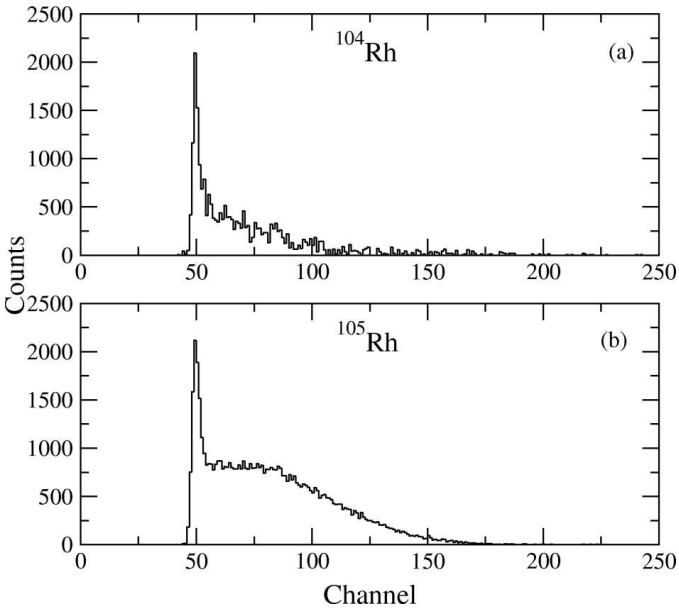

FIG. 2: The $\alpha$-particle spectra detected in coincidence with $\gamma$-rays emitted by ${ }^{104} \mathrm{Rh}$ and ${ }^{105} \mathrm{Rh}$. The contribution of higher energy $\alpha$ particles emitted in the case of ${ }^{105} \mathrm{Rh}$, supporting the hypothesis of the presence of incomplete fusion in its production.

is directly related to the $\gamma$-transition intensities[7]. The simulated and experimental values are shown in Table I. The simu- lated values for the palladium isotopes are in good agreement with the experimental results, suggesting complete fusion as the main reaction mechanism. The simulated values for the rhodium isotopes indicate the presence of another mechanism.

\begin{tabular}{|l|l|l|l|l|}
\hline & $R_{\text {sim }}^{0^{\circ} / 63^{\circ}}$ & $R_{\text {exp }}^{0^{\circ} / 63^{\circ}}$ & $R_{\text {sim }}^{63^{\circ} / 117^{\circ}}$ & $R_{\text {exp }}^{63^{\circ} / 117^{\circ}}$ \\
\hline${ }^{104} R h$ & $0.32(5)$ & $0.74(8)$ & $0.91(18)$ & $1.58(16)$ \\
\hline${ }^{105} R h$ & $0.32(5)$ & $0.74(8)$ & $0.91(18)$ & $2.52(19)$ \\
\hline${ }^{107} P d$ & $0.33(5)$ & $0.32(4)$ & $1.69(34)$ & $1.14(10)$ \\
\hline${ }^{108} P d$ & $0.33(5)$ & $0.32(4)$ & $1.69(34)$ & $1.14(10)$ \\
\hline
\end{tabular}

TABLE I: Simulated and experimental yield ratios for the residual nuclei produced in the ${ }^{11} \mathrm{~B}+{ }^{100}$ Mo reaction.

\section{b) $\mathrm{The}{ }^{16} \mathrm{O}+{ }^{51} \mathrm{~V}$ reaction}

In the ${ }^{16} \mathrm{O}+{ }^{51} \mathrm{~V}$ reaction, the experimental cross-sections for most of the products of this reaction $\left({ }^{60,61} \mathrm{Ni}\right.$ and $\left.{ }^{61,62} \mathrm{Cu}\right)$ are in good agreement with those predicted by the code PACE. The proton and $\alpha$-particle spectra suggest there is no contribution of incomplete fusion in the production of most of the residual nuclei at $70 \mathrm{MeV}$. The contribution of incomplete fusion was observed in this reaction at a higher energy $(90 \mathrm{MeV})[8]$.

In the case of the nickel products $\left({ }^{60} \mathrm{Ni}\right.$ is a $\alpha p 2 n$ channel and ${ }^{61} \mathrm{Ni}$ is a $\alpha p n$ channel), the analysis of the energy spectra of the $\alpha$-particles suggest the presence of higher energy particles in the production of ${ }^{61} \mathrm{Ni}$ (see Figure 3). No difference was found for $\alpha$-particle energies associated with the copper products ( $\alpha$ xn channel) (see Figure 4$)$.

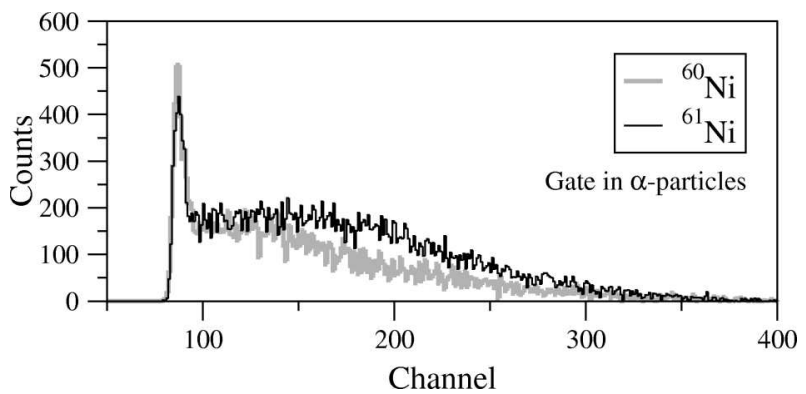

FIG. 3: $\alpha$-particle spectra detected at $0^{\circ}$ in coincidence with $\gamma$-rays emitted by ${ }^{60} \mathrm{Ni}$ and ${ }^{61} \mathrm{Ni}$. There is a contribution of higher energies $\alpha$-particles in the ${ }^{61} \mathrm{Ni}$ spectrum.

In Figure 5 the yield ratios in nickel and copper products for $\gamma$-transitions in coincidence with $\alpha$-particles detected at $0^{\circ}$ and $63^{\circ}$ are shown. Even with the hypothesis of complete fusion mechanism as the main, if not the only, mechanism responsible for population of these nuclei by this specific reaction, different yield ratios for selected transitions were obtained. This indicates that the presence of incomplete fusion is not a necessary condition to have different yield ratios, at least for systems with $\mathrm{A}_{i} 70$.

The simulated yield ratios for this reaction are shown in Table II. The proton gated simulated values for the nickel isotopes are in a good agreement with the experimental results. 


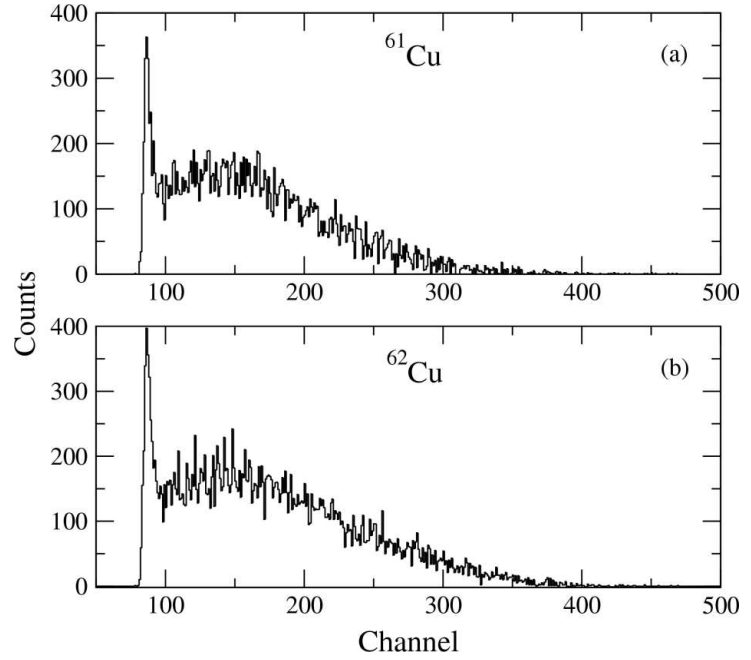

FIG. 4: $\alpha$-particle spectra detected at $0^{\circ}$ in coincidence with ${ }^{61} \mathrm{Cu}$ $\gamma$-rays (a) and ${ }^{62} \mathrm{Cu}(\mathrm{b})$. The spectra indicate that there is no contribution of more energetic $\alpha$-particles.

However, the $\alpha$-gated simulated values suggest the presence of incomplete fusion in both ${ }^{60,61} \mathrm{Ni}$. For the copper isotopes, the experimental yield ratios indicate the presence of another reaction mechanism for ${ }^{61} \mathrm{Cu}$ and ${ }^{62} \mathrm{Cu}$ nuclei.

\begin{tabular}{|c|c|c|c|c|}
\hline & $R_{\text {sim }}^{0^{\circ} / 63^{\circ}}$ & $R_{\text {exp }}^{0^{\circ} / 63^{\circ}}$ & $R_{\text {sim }}^{63^{\circ}} / 117^{\circ}$ & $R_{\text {exp }}^{63^{\circ}} / 117^{\circ}$ \\
\hline${ }^{60} \mathrm{Ni}(\alpha$ gated $)$ & $0.47(7)$ & $1.09(2)$ & $1.91(29)$ & $5.18(13)$ \\
\hline${ }^{60} \mathrm{Ni}(\mathrm{p}$ gated $)$ & $0.35(2)$ & $0.48(4)$ & $1.86(28)$ & $1.84(13)$ \\
\hline${ }^{61} \mathrm{Ni}(\alpha$ gated $)$ & $0.47(7)$ & $0.93(2)$ & $1.91(29)$ & $3.67(8)$ \\
\hline${ }^{61} \mathrm{Ni}(\mathrm{p}$ gated $)$ & $0.35(2)$ & $0.48(4)$ & $1.86(28)$ & $1.61(13)$ \\
\hline${ }^{61} \mathrm{Cu}$ & $0.47(7)$ & $1.39(5)$ & $1.91(29)$ & n.o. \\
\hline${ }^{62} \mathrm{Cu}$ & $0.47(7)$ & $0.89(4)$ & $1.91(29)$ & n.o. \\
\hline
\end{tabular}

TABLE II: Simulated and experimental yield ratios for the residual nuclei produced in the ${ }^{16} \mathrm{O}+{ }^{51} \mathrm{~V}$ at $E_{\text {beam }}=70 \mathrm{MeV}$ (n.o. $=$ not observed).

\section{DISCUSSION}

In the two reactions analyzed $\left({ }^{11} \mathrm{~B}+{ }^{100} \mathrm{Mo}\right.$ and $\left.{ }^{16} \mathrm{O}+{ }^{51} \mathrm{~V}\right)$, yield ratios for $\gamma$-transitions in coincidence with different angles were extracted. Earlier work[2] suggested, as a first step, that the contribution of different reaction mechanisms (complete and incomplete fusion), in the production of residual nuclei, favors the presence of different yield ratios. This difference was seen for the rhodium products measured in the first reaction, and presumed due to incomplete fusion, while there is no difference in the yield ratios for palladium products. However, the results of the analysis of the ${ }^{16} \mathrm{O}+{ }^{51} \mathrm{~V}$ reaction showed it was possible to obtain different yield ratios for different isotopes, even when these nuclei are populated by the same reaction mechanism. This fact can be related to the relatively low recoil velocity of the heavy compound system $\left({ }^{111} \mathrm{Ag}\right)$ of the first reaction $\left({ }^{11} \mathrm{~B}+{ }^{100} \mathrm{Mo}\right)$. Un-
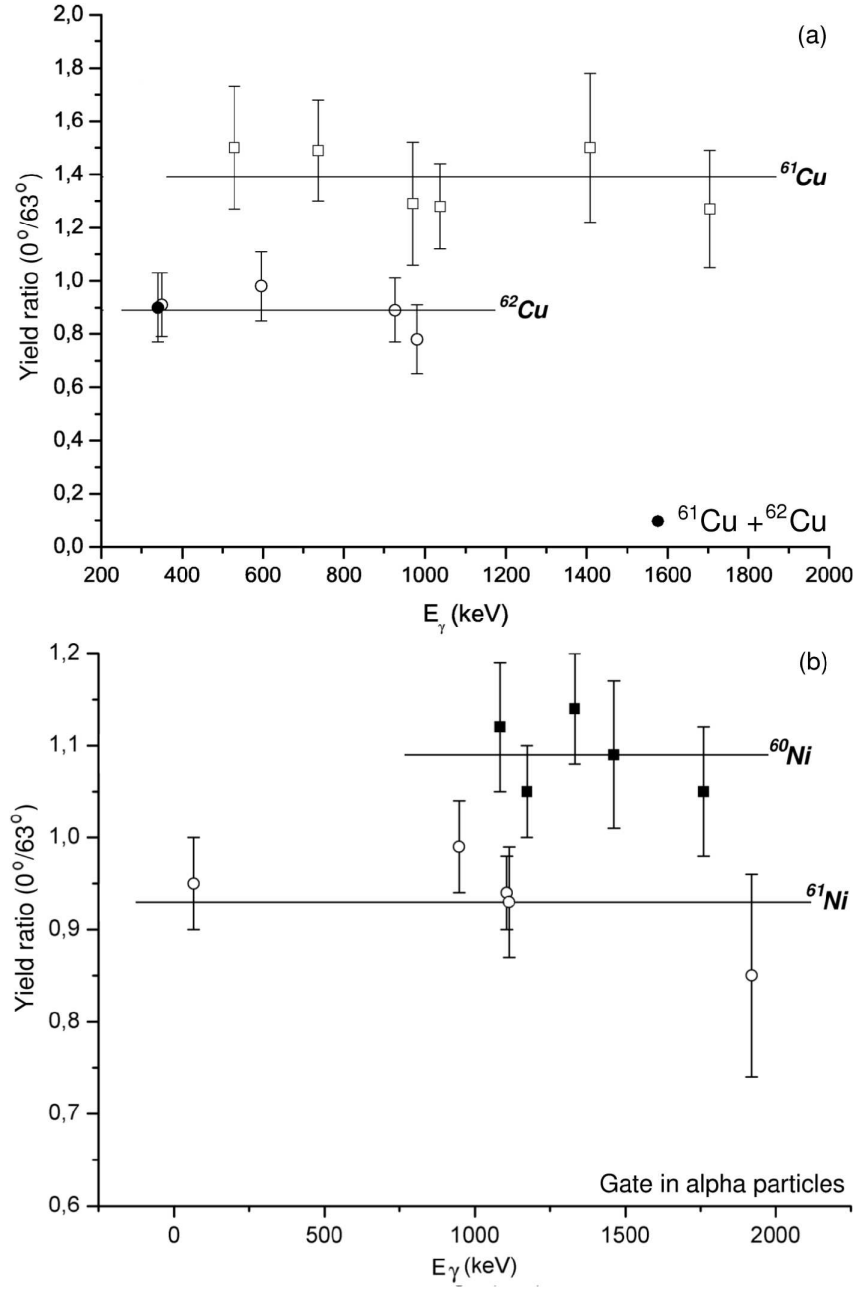

FIG. 5: The yield ratios for selected transitions in (a) copper and (b) nickel products as a function of $\gamma$-ray energy. The yield ratios in nickel products ( $\alpha$ pxn channels) were extracted in coincidence with $\alpha$-particles.

der this condition, if the only mechanism present is complete fusion, the angular distribution of the emitted particles is approximately isotropic even in the laboratory frame, and the presence of incomplete fusion may be a determinant factor in obtaining different yield ratios, because of the enhancement of the anisotropy of the emitted particles, as observed in the case of the rhodium products. In the second reaction analyzed $\left({ }^{16} \mathrm{O}+{ }^{51} \mathrm{~V}\right)$, the compound system is much lighter $\left({ }^{66} \mathrm{Ga}\right)$, so the mass of the compound system was not a limitation, meaning that incomplete fusion was not a necessary condition for obtaining different yield ratios. Although constant yield ratios are drawn on the figures, a simple constant relationship (or even a linear relationship) between yield and $\gamma$-ray energy would not necessarily be always expected, because the transitions analyzed are sampling particular regions of excitation energy. 


\section{CONCLUSION}

The $\gamma$-ray yield ratios were extracted in two heavy-ion reactions for eight isotopes. Different yield ratios were obtained for different isotopes studied even when the reaction mechanism is the same for both (or more) isotopes. The differentiation of the candidate $\gamma$-transitions can be a useful tool for assigning individual transitions to specific isotopes produced in fusion reactions. New experiments are being planned to increase the range of masses studied.

The authors would like to acknowledge financial support by Fundao de Amparo à Pesquisa do Estado de São Paulo (FAPESP) and Conselho Nacional de Desenvolvimento Científico e Tecnológico (CNPq), Brazil.

\section{References}

[1] A. Gavron, Phys. Rev. C 21, 230 (1980).

[2] G.D. Dracoulis et al., J. Phys. G: Nucl. Part. Phys. 23, 1191 (1997).

[3] J.A. Alcántara-Núñez et al., Nucl. Instr. Meth. A 497, 429 (2003).

[4] J.A. Alcántara-Núñez et al., Phys. Rev. C 69, 024317 (2004).

[5] J.A. Alcántara-Núñez et al., Braz. J. Phys 34, 1005 (2004). [6] M.A.G. Silveira et al., Proceedings of the V Latinamerican Symposium on Nuclear Physics, Santos, Brazil, 67 (2003).

[7] K.T. Wiedemann, Master Degree Thesis, IFUSP (2005), unpublished.

[8] S. Mukherjee et al, Eur. Phys. J. A 12, 199 (2001). 